\title{
Lidil
}

Revue de linguistique et de didactique des langues

\section{Le Rapport de stage et la distanciation par rapport à soi}

Mirna Velcic-Canivez

\section{(2) OpenEdition}

\section{Journals}

Édition électronique

URL : http://journals.openedition.org/lidil/19

DOI : $10.4000 /$ lidil. 19

ISSN : 1960-6052

\section{Éditeur}

UGA Éditions/Université Grenoble Alpes

\section{Édition imprimée}

Date de publication : 1 décembre 2006

ISBN : 2-914176-15-5

ISSN : $1146-6480$

\section{Référence électronique}

Mirna Velcic-Canivez, « Le Rapport de stage et la distanciation par rapport à soi », Lidil [En ligne],

34 | 2006, mis en ligne le 07 avril 2008, consulté le 30 avril 2019. URL : http://journals.openedition.org/ lidil/19 ; DOl : 10.4000/lidil.19

Ce document a été généré automatiquement le 30 avril 2019.

(c) Lidil 


\title{
Le Rapport de stage et la distanciation par rapport à soi
}

\author{
Mirna Velcic-Canivez
}

Le problème de l'objectivité

1 Parmi les remarques critiques que nous faisons en évaluant les rapports de stage, l'une des plus fréquentes est, sans doute, celle qui dénonce "un manque de recul», « un manque de distance ». Cette critique signifie qu'un simple compte rendu d'activités ou de tâches ne suffit pas. Il ne suffit pas de "présenter » une organisation ou de décrire la situation professionnelle rencontrée, d'énumérer les objectifs et de préciser la (ou les) mission(s), de signaler les difficultés rencontrées. Il faut montrer que l'auteur du rapport (ou du mémoire) de stage adopte non seulement un langage mais aussi un « raisonnement » professionnel. Et pour raisonner comme un professionnel, il faut savoir prendre de la distance par rapport à l'expérience, à la situation professionnelle. Les critiques que nous adressons à nos étudiants à propos de leurs écrits se justifient donc par le besoin d'atteindre l'objectivité qui seule donne accès à la réalité des pratiques professionnelles.

2 Or nos exigences pédagogiques semblent être difficiles à satisfaire dans la pratique, notamment sur le plan de la rédaction. Le principal obstacle réside dans le fait que le rapport de stage s'appuie sur une expérience personnelle, c'est-à-dire à la fois subjective et singulière. D'un côté, il s'agit de rendre compte en termes objectifs d'une expérience subjective. Mais comment le/la stagiaire peut-il/elle prendre du recul par rapport à cette réalité qui l'affecte dans sa maturation professionnelle et personnelle? L'effort vers l'objectivité l'oblige à une double distanciation: il lui faut se distancier non seulement d'un lieu de stage, d'une mission ou d'un projet pour en faire un objet professionnel, mais aussi de sa propre implication dans la situation professionnelle en question. D'un autre côté, le destinataire d'un écrit professionnel n'est pas un auditeur ou un lecteur naïf qui découvre une réalité professionnelle, c'est-à-dire, qui apprend l'existence d'une situation comme on prend connaissance d'une nouvelle information. Au contraire, qu'il s'agisse d'un commanditaire, d'un tuteur ou d'un évaluateur-membre de jury, le destinataire est un auditeur ou un lecteur instruit, voire savant et, par conséquent, susceptible de partager 
avec le rapporteur le même langage professionnel, les mêmes connaissances, les mêmes valeurs, la même déontologie.

Parmi ces connaissances et ces croyances, il y a un système de stratégies discursives que partagent les interlocuteurs engagés dans la production des représentations professionnelles. Ces représentations sont des modèles des activités ou des situations professionnelles. Ces modèles sont subjectifs et singuliers mais ils constituent un savoir commun (the current knowledge) ainsi que les points de vue que partagent les professionnels à propos d'une situation professionnelle type (Van Dijk, 1994). A côté donc des expériences et des opinions personnelles, ces modèles incarnent un savoir (stéréotypique), les attitudes types qu'il faut partager pour agir et pour parler en tant que professionnel de la communication. Les cognitivistes utilisent le terme "script » pour désigner ces modèles de représentation des situations de vie et de discours que l'on expérimente à répétition, telles que par exemple, voyager ou faire des courses (Tulving, 1995). C'est ainsi que sont assurées la communication et la compréhension à l'intérieur d'une collectivité. Dans cette optique, communiquer une expérience professionnelle n'est pas transmettre des informations. C'est un acte orienté vers le partage des expériences avec autrui.

Il en découle qu'un certain type de destinataire est visé par le rapport de stage. Ce destinataire est un « connaisseur » des différents types de situations professionnelles. S'il est censé apprendre quelque chose, c'est comment le rapporteur-stagiaire témoigne d'une situation particulière, comment il est affecté par la réalité dans laquelle il a été amené à être sujet d'expérience. C'est pourquoi le rapport de stage peut être assimilé à un témoignage. Plus précisément : c'est pourquoi le rapporteur a souvent recours à ce que nous avons identifié comme « mode témoin » dans un autre travail (Velcic-Canivez, 2006). Mais ici apparaît une autre contrainte, une autre dimension de la distanciation par rapport à soi qu'implique l'objectivité. Parce qu'il s'adresse à un destinataire instruit, le rapporteur doit se faire reconnaître comme un «membre qualifié » de la communauté des professionnels.

5 En un mot, l'objectivité du discours professionnel suppose une distanciation par rapport à soi sur laquelle pèse une double contrainte. Le rédacteur d'un rapport de stage doit se distancier du sujet de l'expérience qu'il est aussi. Il doit présenter une expérience professionnelle comme si elle était faite par un autre (que) lui. Il doit aussi présenter une expérience singulière de telle façon qu'elle soit crédible, c'est-à-dire «validable » par la communauté des professionnels.

6 L'objectif de cet article est d'examiner l'un des moyens linguistiques par lesquels les rédacteurs de rapports de stage affrontent cette double contrainte qui provoque une tension au sein du sujet : tension entre la singularité et la subjectivité de l'expérience, d'une part, l'objectivité du rapport et sa validité générale pour la communauté des professionnels, d'autre part. Ce moyen linguistique et textuel est l'emploi du pronom on en alternance avec le je autobiographique.

Méthode et cadre théorique

7 Notre méthode relève d'une pragmatique des textes qui croise la théorie des actes de langage et une théorie du destinataire inspirée de la conception bakhtinienne du langage. Ce deuxième élément méthodologique est lié à la prise en compte du rapport de stage comme genre textuel. Dans une perspective bakhtinienne, en effet, le genre ne représente pas une instance supérieure au texte, instance purement abstraite. Il est caractérisé en premier lieu par la spécificité d'un mode d'énonciation, c'est-à-dire, par une relation 
particulière entre les interlocuteurs. Nous avons l'habitude de limiter l'énonciation à une seule relation : celle qui lie le locuteur à son énoncé. Or nous savons, grâce à la théorie des actes de langage, que les actes illocutoires orientent le sens de l'énoncé différemment. Concrètement, le même passage dans un journal intime et dans une lettre d'adieu invite à des réponses différentes. Autrement dit, parce que le contexte est dans le texte, les énoncés sont des actes de langage. Et parce que les actes de langage obligent à l'anticipation d'une réponse qui valide ce qui se dit, l'énonciation est déterminée par une double relation : il y a une relation qui lie le locuteur à son énoncé ; il y a aussi le rapport qui lie le locuteur à un auditeur intégré par l'énoncé.

8 Cet auditeur se définit, comme le pensait Bakhtine, en fonction du «fond aperceptif présupposé d'un interlocuteur du discours» (1984: 306). Cette instance complique la notion d'acte et les principes mêmes de l'interaction car le destinataire intervient au moment même de l'engagement du sujet dans l'acte de langage. Il s'agit pour celui qui parle, tout comme pour celui qui répond, d'identifier cette instance responsive. Il s'agit de se l'approprier pour savoir parler et pour savoir répondre puisque nous écoutons et parlons non seulement en fonction de nos intentions et de nos connaissances, mais surtout pour nous affirmer en tant que partenaires crédibles. En somme, le destinataire ne peut pas correspondre à un simple partenaire coopératif. Dès lors qu'il s'agit d'un interlocuteur intégré par le texte, la validation de ce qui se dit ne peut pas être fondée sur la symétrie ou sur la soumission du destinataire au locuteur. Le partenaire n'est pas temporellement second dans le dialogue car c'est lui qui reconnaît ou pas la crédibilité du propos. De ce fait, il est possible de dire que c'est, au contraire, le locuteur qui est tenu par (l'idée de) celui à qui il adresse ses énoncés. Dans cette perspective, les relations d'interlocution, tout comme les relations d'interaction, se conçoivent sur le plan de la reconnaissance. Les rapports de stage en sont un exemple particulièrement clair : les rapporteurs visent un destinataire qui sache reconnaître le caractère professionnel de ce genre d'écrits'.

Le je autobiographique

9 Les passages autobiographiques sont importants dans la construction d'un sujet professionnel distancié de la réalité du stage proprement dite. Le rapporteur a besoin de ce recul pour s'affirmer dans sa fonction de rédacteur. Ces passages autobiographiques se limitent souvent à des commentaires stéréotypés, tels que, par exemple: ce stage a "enrichi mes connaissances", ce stage a "ouvert de nouveaux horizons devant moi". Néanmoins, il est possible de citer des extraits plus élaborés qui insistent sur le caractère personnel d'une expérience professionnelle :

(1) «Communiquer est un enjeu majeur pour les institutions publiques. La mairie dispose de beaucoup de moyens pour réaliser des actions de communication. L'évènementiel [...] est très apprécié. La communication écrite se porte bien. [...]. Pour être sincère, je suis arrivée en stage pensant que la communication en mairie aurait un rythme plutôt tranquille. Or la situation est en réalité tout autre : il y a énormément de travail et le rythme des journées est très soutenu ». ).

10 L'exemple est révélateur par la marque : pour être sincère qui représente la transition d'un passage descriptif, rédigé à la troisième personne, à une partie clairement autobiographique : le sujet se présente comme affecté dans sa manière de voir certains secteurs professionnels. Le rapporteur essaie de prendre du recul par rapport à une réalité professionnelle en se thématisant en tant que sujet d'expérience : je suis arrivée en 
stage pensant que [...]. L'exemple suivant, par contre, met en scène directement les sentiments de la stagiaire :

(2) «J'aurais aimé pouvoir plus aider à la réalisation d'un dossier de presse, mais deux ou trois personnes travaillaient déjà dessus et j'aurais certainement été de trop ».

Rapport de stage d'Aurélie M. (stage à la SNCF dans un service de communication, juin 2005).

11 Le rapporteur exprime le regret de ne pas avoir pu s'imposer au groupe en tant que sujet d'expérience et mettre à profit ses compétences. L'indice ou la marque de distanciation de ses activités de stage et de soi change : le recours à un conditionnel passé (J'aurais aimé...) est ici révélateur. Mais avant tout, ces exemples démontrent que la distanciation d'une réalité professionnelle et, en même temps, une relation avec soi n'est pas facile à effectuer ni sur le plan de la rédaction proprement dite, ni d'ailleurs sur le plan de la théorie de l'énonciation. Ce phénomène concerne directement le statut référentiel et textuel du je autobiographique : le phénomène de distance met en évidence la complexité de la référence $d u$ « je » autobiographique, complexité occultée par la première personne grammaticale. C'est pourquoi ces «ilots autobiographiques" au sein d'une écriture professionnelle invitent à une analyse plus attentive de cet indice décisif dans la construction des modèles de soi.

Sujet autobiographique identique à soi : coïncidence du rapporteur et du stagiaire

12 La première personne désigne, on le sait, le locuteur, la première personne du discours. Dans le régime autobiographique, elle a la propriété de renvoyer à un sujet identique à soi, auquel cas celui qui parle et celui dont on parle - le locuteur et le sujet d'expérience sont identifiés comme une seule et même personne. L'extrait suivant d'un rapport de stage peut être éclairant sur ce point :

(3) « J'ai entrepris de réaliser un fichier clients ainsi qu'un fichier prospects [...]. J’ai également initié l'uniformisation de l'identité visuelle de l'agence en créant une signature commune à tous les mails. Dans le même temps, j'ai réfléchi à la création d'un support [...]. Dans ce but, j'ai imaginé un document [...]. Enfin, dans l'objectif de faire connaître l'agence à l'extérieur, j'ai initié une campagne de relations presse [...].

En partant de ce principe, j'ai rédigé un communiqué qui permet de comprendre ce que fait l'agence [...]. J'ai par la suite cherché les coordonnées des journalistes sur les sites Internet. [...] Enfin, j'ai eu l'occasion de rencontrer quelques clients [...] ».

Rapport de stage de Olivia M. (stage dans une agence de communication évènementielle, juin 2006).

13 Dans ce passage, la première personne renvoie à un sujet autobiographique identique à soi : le rapporteur coïncide avec le sujet d'expérience. Il y a fusion entre la réalité du stage et le discours du rapport. De plus, on note que le je autobiographique se combine systématiquement avec des verbes au passé composé, lequel a le pouvoir de mettre le procès en relation avec l'actualité de l'acte même de l'énonciation. Le procès au passé composé est présenté comme antérieur, ponctuel, aoristique mais dépendant de ses conséquences ultérieures. Par ailleurs, la forme composée de ce temps verbal entraine l'aspect accompli. D'où l'importance de ses valeurs aspectuelles qui peuvent, dans certaines circonstances, s'imposer aux valeurs temporelles. C'est le cas de la plupart des procès de ce passage qui s'interprètent comme des résultats, comme des accomplissements. Certains arguments syntaxiques dans la configuration des prédicats peuvent confirmer cette interprétation: les verbes sont accompagnés soit d'un objet direct au singulier (j'ai imaginé un document, j'ai rédigé un communiqué, j'ai eu l'occasion), soit 
d'un complément prépositionnel qui comporte lui-même un objet direct au singulier (J'ai entrepris de réaliser un fichier, j'ai réfléchi à la création d'un support). Autrement dit, les procès antérieurs (ou situés dans le passé) et le sujet de l'expérience qui en fait partie ne sont pas décalés de l'actualité de l'énonciation. Le discours du rapporteur ne se détache pas de la perspective du sujet d'expérience. Le sujet autobiographique représenté dans de ce passage ne donne pas lieu à une distanciation de soi². Par conséquent, le destinataire ne peut pas distinguer clairement où se termine l'univers de la personne impliquée dans une situation professionnelle proprement dite et où commencent l'univers du rapporteur et son discours. En d'autres termes, il ne peut pas savoir où se termine l'expérience liée à un lieu de stage et où commence le commentaire de cette expérience. Ce sujet identique à soi s'impose en tant que sujet impliqué dans une série d'accomplissements. Cela a des conséquences sur la structure textuelle de ce rapport de stage et sur le plan de l'énonciation: le rapport s'organise selon la logique d'une énumération et se constitue comme un compte rendu d'activités.

Sujet autobiographique non identique à soi : non-coïncidence du rapporteur et du stagiaire

En revanche, dans le discours autobiographique d'un autre rapport de stage, nous retrouvons une distanciation à l'égard de soi et d'une réalité de stage. Le passage a l'aspect d'un commentaire :

(4) « ... j'ai appris un vrai métier d'organisateur, de gestionnaire et de créateur dans la communication. Si bien des doutes m'ont envahi au sujet de mon avenir, je suis désormais rassuré. Non pas que je sache que mon avenir se situe précisément dans l'évènementiel, mais je sais maintenant que je suis capable d'être un vrai professionnel de la communication dans une grande entreprise. J'ai trouvé dans ce stage les outils mais surtout la confiance et la motivation pour réaliser une action dont je suis fier ».

Rapport de stage de Timothée L. (stage dans le service de communication d'une entreprise moyenne, juin 2005).

On note que, dans ce passage, le je est associé à des verbes ayant un sens statique, principalement au verbe "être " - verbe d'état par excellence - et à des verbes qui dénotent des activités intellectuelles inséparables de la personne. Ces verbes sont systématiquement au présent (je suis désormais rassuré,je sais maintenant que je suis capable, je suis fier). Le passé composé a la valeur aspectuelle d'un présent accompli (j'ai appris, J'ai trouvé). Ce sujet autobiographique ne peut pas être confondu avec le sujet d'expérience car les procès auxquels je est associé en tant que sujet grammatical se situent dans le présent. C'est pourquoi son référent renvoie clairement au rapporteur qui présente ses expériences comme antérieures à la rédaction du rapport. Dans ce passage, le rapporteur quitte, en quelque sorte, le lieu de stage et se projette dans un avenir professionnel. Il se détache de l'univers d'un étudiant-stagiaire pour se présenter comme une personne qui s'inquiète de ses compétences professionnelles, qui est habitée par des doutes. Le je autobiographique renvoie à un rapporteur qui ne peut pas être confondu avec le sujet d'expérience : il est clairement décalé par rapport à la personne impliquée dans une pratique professionnelle. D'où le statut de commentaire de ce passage autobiographique. Le commentaire porte sur le thème (ce stage) antérieurement développé sous forme de récit d'expérience. Le sujet de ce passage est clairement identifié comme un commentateur, comme la personne du rapporteur à qui l'on peut attribuer la responsabilité de ce qui se dit. 
16 La personne du rapporteur ne peut pas être ici confondue avec le sujet d'expérience faisant partie du thème antérieurement développé. Le sujet autobiographique est non identique à soi. Il est comme dédoublé, ce qui permet au rapporteur de se détacher du stagiaire et, par conséquent, de mieux insister sur le caractère véridique de son expérience afin de la valoriser. La répétition de vrai pour qualifier un savoir-faire professionnel (un vrai métier d'organisateur) ou une vocation (être un vrai professionnel de la communication) est ici symptomatique.

17 Les exemples (3) et (4) démontrent clairement que le je autobiographique peut véhiculer plusieurs modèles de soi. Le sujet autobiographique est un sujet à plusieurs dimensions. Ni le recours à la première personne ni le passage au régime autobiographique au sein d'un rapport de stage n'entrainent automatiquement la prise de recul. Le régime autobiographique ne garantit pas la distanciation par rapport à soi, ce qui est nécessaire pour rendre une expérience professionnelle « objective ». Il permet, certes, d'exprimer la singularité de cette expérience. Mais une expérience professionnelle ne peut pas être seulement singulière. Pour être validée, nous l'avons dit, elle doit permettre une relation de partage; elle doit être reconnue par un destinataire ayant lui-même vécu des situations semblables. Une expérience professionnelle est censée être généralisable, elle peut être reconnue dans sa singularité à condition d'être subjective et générale.

18 Néanmoins, le fait d'insister sur l'idée de partage pose d'autres problèmes: comment établir une relation de partage avec un interlocuteur instruit mais qui est une autre personne et qui est, de ce fait, susceptible d'avoir des expériences qui diffèrent de celles qu'exprime le rapporteur? Quels sont les moyens proprement linguistiques aptes à introduire, sur le plan de l'énonciation, une telle relation dialogique?

L'alternance je autobiographique/on

L'exemple suivant est marqué par la généralisation des expériences singulières :

(5) « \e ne voyais mon maître de stage qu'une fois par semaine pour faire le point sur mon travail. [...]. J'avais une assez grande liberté dans mes actions et démarches. C'est formateur d'être autonome car on apprend à se débrouiller par soi-même, à faire ses propres choix, en somme, à être responsable [...].

Le plus difficile n'a pas été la réalisation du travail mais tout le temps de préparation: récolter les différentes informations, nombreuses personnes à voir, lire et synthétiser de nombreux documents ",

Rapport de stage de Sophie V. (stage dans une agence de communication, juin 2006).

20 Le passage se structure en alternant une énonciation à la première personne et une énonciation à la troisième personne. Celle-ci est actualisée à l'aide du pronom on.

On $=$ je + quiconque se trouvant dans une situation professionnelle semblable

21 Le pronom on se manifeste ici dans sa valeur la moins personnelle : il désigne je + la communauté discursive en question, c'est-à-dire, quiconque se trouvant dans une situation professionnelle semblable ${ }^{3}$. Cette valeur du on permet d'inclure le référent du je, c'est-à-dire, le stagiaire, dans une communauté de personnes ayant des expériences similaires. Il s'agit d'une communauté formée par n'importe quels étudiants-stagiaires. Cette généralisation de l'expérience est maintenue par le présent du verbe : on apprend à [...]. Cependant il se produit un changement de statut de l'énoncé comportant le on : l'énoncé on apprend [...] ne se situe pas sur le même plan textuel que les énoncés actualisés en régime autobiographique. Le changement de plan est introduit, bien entendu, à l'aide du démonstratif dans c'est [...] qui est, comme nous le savons, un indice de rupture. Dans ce 
contexte, ce démonstratif est également un démonstratif de reprise. De ce fait, il signale un décalage énonciatif en reformulant le thème du passage - «l'autonomie professionnelle »-, en le réintroduisant sur un autre angle. Ce décalage énonciatif est accusé par le changement de la personne du sujet (je/on) et par le temps du verbe (imparfait/présent) dans la coordonnée. Par conséquent, l'expérience associée à un sujet représenté par je est séparée de l'expérience associée à un sujet représenté par on. Les expériences singulières de la stagiaire sont présentées en décalage par rapport aux expériences semblables des professionnels d'une communauté particulière (stagiaires). Ainsi, la transition de je à on marque un écart entre l'antériorité d'une expérience singulière et l'actualité des expériences semblables : le discours du rapporteur se détache $\mathrm{du}$ discours du stagiaire actualisé en régime autobiographique. Il est clair qu'en se détachant ainsi de la singularité de son expérience et, par conséquent, en se distanciant de soi, le rapporteur fait valoir son expérience de stagiaire en tant qu'expérience professionnelle: le passage se lit comme le commentaire de la partie antérieure, réalisée à la première personne. Est donc visé un destinataire qui serait prêt à reconnaître le caractère professionnel du stage, le professionnalisme du stagiaire.

On = je + quiconque prêt à partager certaines positions à partir d'une situation professionnelle semblable

Néanmoins, le maniement du pronom on exige une certaine prudence. Nous savons que la valeur de base de on est celle d'un pronom indéfini : son référent n'est jamais une pluralité d'individus déterminés. C'est pourquoi il peut fonctionner comme « substitut de tous les autres pronoms personnels en rejetant leur référent dans l'anonymat » (Riegel et al., 1996 : 197). Il y a donc un risque de dépersonnalisation de l'expérience.

Pourquoi prendre ce risque? Il semble en effet que ce soient les valeurs les moins personnelles qui sont les plus fréquentes dans le contexte de la généralisation des expériences singulières. L'exemple suivant permet d'apporter des précisions à cette observation:

(6) J'ai été surprise de constater que chez ETDE les employés ne restent pas très longtemps.

On remarque rapidement que tout le monde se tutoie ou presque, [...]. On s'appelle par son prénom. On évite les «monsieur ». On se parle très franchement, sans manière, que tout aille bien ou non. Ce n'est pas une habitude que l'on trouve au sein de toute entreprise. J'en déduis que «les barrières" qui pourraient s'instaurer «naturellement » entre la direction et les employés ne sont pas ici présentes. [...] En somme, le rapport à l'autorité n'est pas très marqué dans la façon dont les personnes communiquent entre elles. Mais est-ce vrai pour autant que chacun ne sait pas où est « sa place »?

Rapport de stage de Sophie V. (stage dans une agence de communication, juin 2006).

Le premier on est associé à un verbe dit d'opinion (on remarque). Ces verbes permettent d'introduire dans le discours une prise de position. Associé aux verbes d'opinion, on désigne quiconque est prêt à partager certaines positions à partir d'une situation professionnelle semblable. Son référent est donc je +la communauté discursive en question, c'est-à-dire le stagiaire et n'importe quel professionnel qui partage la même prise de position. Ces sujets d'expérience seraient en même temps les « observateurs » de leurs pratiques, ceux qui réfléchissent sur leurs actions. Ainsi ils seraient dotés d'une qualité supplémentaire qui consiste précisément à savoir prendre du recul. Ces sujets seraient dotés d'un savoir-faire spécifique, d'une compétence propre à n'importe quel rapporteur-stagiaire apte à se distancier de soi afin de prendre ou de partager des 
positions professionnelles. Ainsi, le on dans on remarque permet d'inclure non seulement le stagiaire mais aussi le rapporteur dans une communauté de professionnels ayant des expériences semblables. Le destinataire visé est celui qui serait prêt à reconnaître la pertinence professionnelle du stage et, surtout, le professionnalisme du rapporteur, sa compétence de se détacher de la réalité du terrain pour mieux la rendre dans l'écrit.

On $=$ ils

Suit une série de on dont la valeur change : le on dans On s'appelle [...], On évite [...], On se parle [...] désigne les professionnels à l'intérieur de l'entreprise en question, c'est-à-dire une autre communauté de personnes. Ces occurrences rapprochent le on du ils. La valeur de on est donc ici plus personnelle car son référent vise la personne qui est constitutive de l'objet (d'expérience). Cette personne est clairement "distanciée» de la première personne du discours puisque c'est une non-personne. La première personne est donc exclue du référent du on. Toutefois, ce passage produit une sorte de proximité dont il faut rendre compte.

On note que le pronom on change de valeur si l'on transforme le temps du verbe en mettant les verbes de cette série au passé composé. Dans ce cas, il se produit un glissement de sens du pronom on : son référent prend un sens inclusif, ce qui amène à une rupture discursive dans le passage :

(6a) J'ai été surprise de constater que chez ETDE les employés ne restent pas très longtemps.

On remarque rapidement que tout le monde se tutoie ou presque, [...]. ${ }^{* *}$ On s'est appellé par son prénom. ${ }^{* *}$ On a évité les «monsieur ». ${ }^{* *}$ On s'est parlé très franchement, sans manière, que tout aille bien ou non. [...].

Cette rapide transformation de l'exemple suggère que c'est le sujet on en combinaison avec le verbe au présent qui est à l'origine de l'effet de proximité dans le passages.

Il est connu que le référent de on est indécis car il se trouve "à la frontière » entre la personne et la non-personne, entre les personnes du discours et la personne dont on parle (Atlani, $1984: 26)$. C'est un « pronom transitionnel » entre je et $i l$; il occupe un « no man's land de la personne » (Détrie, 1998: 29). Lorsque, sur le plan de la référence, on s'approche de ils et désigne la " personne-objet ", il faut noter que cette personne-objet n'est pas entièrement autonome. Elle est, du point de vue de l'énonciation et dans une perspective textuelle, dépendante de la relation je-tu. Or le sujet on exclut ici le je du rapporteur-stagiaire en renvoyant à une personne externe à la relation je-tu. Ce qui peut surprendre, c'est qu'en même temps nous sommes amenés à participer, en quelque sorte, à l'atmosphère de cette entreprise. Il y aurait donc, paradoxalement, une proximité de l'expérience. Cet effet se produit sur le plan textuel malgré l'exclusion du sujet autobiographique sur le plan de la référence du on. Notre interprétation est que cette proximité concerne la relation avec la deuxième personne du discours. Le destinataire est " pris à témoin ». Il se manifeste comme un regard qui « colle à la réalité du terrain », ce qui permet de suivre une situation professionnelle depuis l'intérieur de cette situation, de la partager en certains de ses aspects. Cela permet de comprendre, en partie, pourquoi les occurrences de on qui ont la valeur de ils produisent, dans ce passage, à la fois un effet de distance et de proximité : distance au sens d'une extériorité par rapport à soi et proximité au sens d'un rapprochement de la part de la deuxième personne du discours. Le phénomène a nécessairement des conséquences sur le plan textuel: le passage ne se lit plus comme un " commentaire » mais plutôt comme "un témoignage ». Le destinataire visé n'est plus seulement amené à reconnaître le professionnalisme du stagiaire ou du 
rapporteur. Pris à témoin, il est censé (re-)découvrir la réalité du stage comme une expérience singulière et, en même temps, proche de ses propres expériences professionnelles. Par conséquent, il est amené à l'identifier comme « vraie ».

Cette proximité qui s'établit entre un objet d'expérience et le destinataire disparait lorsque le on est remplacé par des termes qui figurent dans le contexte linguistique, c'està-dire soit par un sujet clairement indéfini, tel que le pronom tout le monde :

(6b) J'ai été surprise de constater que chez ETDE les employés ne restent pas très longtemps.

On remarque rapidement que tout le monde se tutoie ou presque, [...]. Tout le monde s'appelle par son prénom. Tout le monde évite les «monsieur ». Tout le monde se parle très franchement, sans manière, que tout aille bien ou non. [...].

soit par un sujet défini et nommé, tel que les employés et qui autorise une reprise par l'anaphorique :

(6c) J'ai été surprise de constater que chez ETDE les employés ne restent pas très longtemps.

On remarque rapidement que tout le monde se tutoie ou presque, [...]. Les employés s'appellent par son prénom. Ils évitent les «monsieur». Ils se parlent très franchement, sans manière, que tout aille bien ou non. [...].

Dans les deux cas le destinataire maintient la distance vis-à-vis des procès sans pouvoir entrer dans une relation de partage. Il n'est pas pris à témoin. Par conséquent, les procèsexpériences de ce passage ne sont pas nécessairement reconnus comme pertinents. Cela a encore une fois des conséquences sur le plan textuel : le passage ne se lit plus comme « un témoignage » mais plutôt comme « une description ».

Si donc le premier on (le moins personnel) généralise les expériences en incluant le sujet dans une communauté de " membres qualifiés » (On apprend à se débrouiller par soi même [... ], On remarque rapidement [...]), le deuxième on qui est proche de ils généralise aussi, mais en établissant une relation de proximité avec le destinataire (On s'appelle, On se parle, etc.). Conclusions

33 Dans une situation de rapport de stage, la tension au sein du sujet - tension entre la subjectivité de l'expérience et l'objectivité du rapport - se résout sur deux plans. Sur le plan de l'énonciation, elle se résout par la relation que le sujet établit avec son destinataire. Celui-ci est impliqué en tant que "connaisseur " (ayant des expériences professionnelles semblables) et en tant que personne (ayant des expériences nonidentiques à celles du sujet parlant). C'est un destinataire qui est censé à la fois reconnaître et découvrir les expériences d'autrui. Ainsi, la relation que le rapporteur de stage établit avec son destinataire est une relation de partage entre des interlocuteurs instruits, voire savants, mais inégalement concernés en tant que personnes ${ }^{6}$.

34 Sur le plan linguistique et textuel, la tension du sujet d'un rapport de stage se résout à l'aide de moyens susceptibles de généraliser les expériences. L'un de ces moyens est le pronom on employé dans ses différentes valeurs et en alternance avec le je autobiographique. Dans des conditions spécifiques, démontrées par notre analyse, le pronom on a le pouvoir de prendre le destinataire à témoin. D'où la principale idée de cet article: c'est en établissant une relation particulière avec le destinataire et par des moyens linguistiques et textuels précis que le rapport de stage construit une forme d'objectivité fondée sur le partage des expériences et non sur un simple compte-rendu factuel'. Le rapport de stage n'est pas un simple exposé de faits, c'est surtout le témoignage d'une expérience professionnelle. 

développé. Les récits de témoignage sont, eux aussi, caractérisés par une double identité : ils doivent satisfaire à la fois des critères d'objectivité et de subjectivité. Ils sont objectifs au sens où ils renvoient à une réalité effective et non pas à une réalité fictive. Cela les rapproche du discours historique dont on attend des connaissances, un savoir valide sur ce qui fut et que l'on appelle "la vérité historique ». En même temps, les récits de témoignage sont des récits d'expérience : ils portent sur une réalité vécue qui fait partie de l'univers d'expérience de la personne qui parle. D'où un rapprochement avec le discours autobiographique et, par conséquent, l'identité hybride de cette catégorie de textes: les récits de témoignage croisent le discours historique et le discours autobiographique car ils sont censés représenter l'objectivité d'une expérience singulière. Parce que le récit de témoignage en tant que catégorie textuelle ou discursive associe l'autobiographique et l'historique, il autorise un parallèle avec le rapport de stage qui croise la subjectivité autobiographique et l'objectivité du discours professionnel.

Tout comme le locuteur d'un rapport de stage, le locuteur-témoin est amené à prendre ses distances par rapport à une réalité qui fait partie de son univers d'expérience, par laquelle il a été directement affecté. Il y a donc un "paradoxe du témoin » : une prise de distance par rapport à soi qui est un mode d'objectivation du vécu personnel, une manière d'en rendre la vérité. Comme dans une situation de rapport de stage, le témoignage oblige à une distanciation du sujet par rapport à une réalité et invite à une distanciation par rapport à soi. Dans les deux cas, il s'agit d'actes et de textes qui portent sur des expériences. Dans les deux cas, il y aurait une double distanciation motivée par un appel à la reconnaissance.

Envisagé comme témoignage d'une expérience professionnelle, le rapport de stage a un avantage cognitif et pédagogique. Le caractère asymétrique de la relation entre les interlocuteurs inégalement concernés permet de redéfinir le processus de communication. Celui-ci, loin de se limiter à la transmission des objectifs, des actions, des démarches, est un processus de transformation des informations. Témoigner ne mobilise pas la compétence de transmettre des informations ; c'est un acte qui nous mobilise dans la capacité de nous interroger sur l'expérience d'autrui et de la partager. Anticiper un auditeur dont le vécu est autre que le mien ou apprendre que des situations semblables peuvent être expérimentées différemment par des personnes différentes permet $d^{d}$ '« ouvrir nos horizons », comme le disent les étudiants-stagiaires. Cela permet d'ouvrir nos horizons dans le sens où ce processus de compréhension passe par l'inadéquation, par les écarts entre les personnes et, de ce fait, par des moments d'incompréhension peutêtre. Ainsi, témoigner d'une expérience professionnelle ne nous incite pas tant à partager les mêmes points de vue qu'à changer nos manières de voir les réalités qui constituent nos expériences.

\section{BIBLIOGRAPHIE}

Charaudeau, P. et MAingueneau, D. (2002) : Dictionnaire d'analyse du discours, Paris, Seuil. 
DELVENNE, S., MiCHAUX, CH. et DOMINICY, M. (2005) : Oralités. Catégoriser l'impensable. La figure du «musulman » dans les témoignages de rescapés des camps nazis, Bruxelles, Université Libre de Bruxelles.

DETRIE, C. (1998) : Entre altérité et ipséité : statut énonciatif de « on » dans Sylvie, L'information grammaticale 76, 29-33.

VELCIC-CANIVEZ, M. (2002) : Le récit de témoignage comme genre. Spécificités référentielles, in M. Ballabriga (éd.), Analyse des discours. Types et genres : Communication et Interprétation, Toulouse, Éditions universitaires du Sud, coll. « Champs du Signe », 317-329.

VELCIC-CANIVEZ, M. (2004) : Les récits de témoignage : expérience indicible ou réception problématique? in D. Salas (éd.), Victimes de guerre en quête de justice, Paris, L'Harmattan (« Sciences Criminelles »), 135-155.

VelCic-Canivez, M. (2006) : Prendre à témoin, Paris, Ophrys.

VAN DIJK, T. (1994) : Discourse and Cognition in Society, in D. Crowley et D. Mitchell (éds.), Communication Theory Today, Oxford, Polity Press/Blackwell Publishers, 107-127.

\section{NOTES}

1. Les exemples dont je me sers proviennent de rapports (mémoires) de stage de deuxième année Infocom et de licence professionnelle Infocom Organisation des entreprises. Ils sont, bien entendu, trop peu nombreux ici pour constituer un véritable corpus. Cependant, les traits repérés sont largement répandus dans ce genre d'écrit et les analyses proposées s'appuient sur des exemples généralisables.

2. Il ne faut pas confondre la distanciation par rapport à soi avec la distance que le passé composé introduit entre le temps raconté et le temps du raconter : celle-ci se justifie par la relation que le passé composé établit entre le point de l'événement et le point de l'énonciation (Bres, 1994, 1998).

3. Voir l'analyse de on dans les articles scientifiques de K. Fløttum (2004). Il n'est pas surprenant que certaines valeurs de on, notamment celles qui sont les moins personnelles et que l'auteur de cet article a pu identifier dans le discours dit « académique » (Fløttum \& Rastier, 2003) puissent être repérées également dans les rapports de stage. Dans les deux cas, il s'agit d'écrits fortement orientés vers la crédibilisation des connaissances et/ ou des expériences.

4. C'est pourquoi ces verbes peuvent être également associés à un sujet en première personne, dans les expressions telles que Je pense que, J'estime que. Ces expressions sont fréquentes dans le discours académique, mais semblent peu convenables dans le contexte des rapports de stage.

5. Il serait intéressant d'analyser les conséquences d'une autre transformation de ce passage, au moyen d'une combinaison de on et de l'imparfait. Mais cela dépasse largement le cadre de cet article. Néanmoins, nous renvoyons à l'article de A. Rabatel (2001), particulièrement éclairant et riche en observations sur la combinaison de on et de l'imparfait dans le contexte d'une analyse menée sur les textes littéraires.

6. C'est précisément la relation avec un tel destinataire - instruit (ou renseigné), mais différemment concerné ou affecté - qui caractérise ce que nous appelons ailleurs « le mode témoin » (Velcic-Canivez, 2006). 
7. Il est clair que cette idée concerne toute une palette de moyens textuels et linguistiques. Il faudrait la vérifier par l'analyse exhaustive de ces moyens appuyée sur l'étude d'un véritable corpus de textes, ce qui dépasse les ambitions de cet article.

\section{RÉSUMÉS}

Le rapport de stage se laisse identifier comme un travail à la fois personnel et professionnel. Il est à la fois subjectif et objectif : subjectif car il porte sur une expérience singulière du terrain ou d'un lieu de stage ; objectif car il est censé présenter une expérience singulière de façon à la faire reconnaître, c'est-à-dire "valider " par la communauté des professionnels. L'effort vers l'objectivité oblige le rapporteur à une double distanciation : il lui faut se distancier non seulement d'un lieu de stage ou d'une pratique pour en faire un objet professionnel, mais aussi de sa propre implication dans la situation professionnelle en question. L'objectif de cet article est d'examiner l'un des moyens linguistiques par lesquels les rédacteurs de rapports de stage affrontent cette double contrainte. Ce moyen linguistique et textuel est l'emploi du je autobiographique en alternance avec le pronom on. L'analyse insiste sur l'importance de la relation avec le destinataire. 